\title{
AdAM CZyszczoń \\ ALEKSANDER ZGRZYWA \\ CONSENSUS AS A TOOL SUPPORTING \\ CUSTOMER BEHAVIOUR PREDICTION IN SOCIAL CRM SYSTEMS
}

Abstract

Keywords
Social Customer Relationship Management systems represent a new area in the field of CRM which together with rapid development of Social Networks and Social Media has acquired strategic importance for many companies. As a response to ongoing challenges related to growing customer expectations, in this paper we present intelligent tools for customer behaviour prediction in Social CRM systems. The use of the consensus approach is aimed at resolving contradictory forecasts of customer behaviour provided by different agents working as independent Artificial Neural Networks systems. The goal of the presented tool is to improve prediction functionality of customer behaviour.

CRM, Social CRM, consensus method, customer behaviour, behaviour prediction 


\section{Introduction}

The advantages of Customer Relationship Management (CRM) systems seem to be significant enough that it is advisable to use these systems to analyse customer behaviour, in particular, in the domain of Social Media - that is in Social CRM (sCRM) systems. The application of such an analysis meets the expectations of customers through better match to their needs. Moreover, the innovation of presented solution may be advocated by the fact that, according to earlier studies conveyed in 2010, no system of among the world leading CRM vendors such as SAP, Oracle, Salesforce.com, and Microsoft, did not have similar functionality. The CRM systems collect information about customer activity and characteristic properties of their profiles. Thanks to this, systems of this type contain sufficient information in order to be able to predict customer behaviour.

The goal of this paper is to present intelligent tools for customer behaviour prediction in Social CRM systems which use a consensus approach in order to resolve different conflict situations. We define conflict situation as contradictory forecasts of behaviour of particular customer or potential customer predicted by different agents. Because predicting customer behaviour is difficult due to many complex conditions which determine it, each prediction uses the Artificial Neural Network (ANN). Predictions are based on chosen historical data of customer profile with the emphasis on social networking information. The aim of the presented tools is to improve prediction functionality of customer behaviour.

\section{Related work}

The key concept of customer behaviour prediction in CRM systems was presented in our previous research [1] where we presented its basic components and developed methods utilizing ANN for behaviour prediction. We also extracted key characteristics of customer profiles and fundamental behaviour factors regarding the data collected by the CRM systems. This included also information on client activity in the sphere of social networking websites. This paper may be considered as a continuation to the previously conveyed research by extending and improving prediction methods.

Another important scientific work is the research carried out by N. T. Nguyen [6, 5] where the author introduces intelligent technologies for the resolution of knowledge inconsistency in various computer systems applications. This research is considered as a theoretical base for resolving conflicts using the consensus system approach.

\section{Customer Relationship Management and Social CRM}

In an increasingly competitive market the struggle for customers using traditional methods may not be very effective. A company which is trying to actively gain a competitive advantage should continuously extend knowledge about its customers. To achieve this companies use the strategy of customer relationship management what can be 
clearly seen in growing interest in this domain in recent years. On the other hand, there are many different interpretations of this concept, therefore it is ambiguous and difficult to define. Frequently this term is used in the context of some technological solutions, in particular CRM systems aimed at identifying and satisfying customer needs and reducing costs. Some, however, treat CRM as a technology supporting the idea of marketing based on relations, in the context of collecting and processing information. Other researchers believe that customer relationship management is a recipe for management focused around the customer and for maintaining a long term relationship with him. Technology in this perspective of CRM concept is first of all understood as technical devices that support and automate some business processes in order to achieve stated objectives more effectively. The author of a book on the subject of CRM [3] has reviewed the various definitions of this concept and extracted from them elements which occur most frequently and were highlighted by their authors as most important. Resulting list of definitions allowed to obtain synthetic picture of the definition of CRM, which is as follows:

"CRM is first of all a philosophy, or business strategy, whereas the tool supporting the realization of this philosophy/strategy becomes the technology of information processing." (translated from [3]).

In this paper we consider Customer Relationship Management as an ongoing process to provide added value to the customer, while a CRM computer system is a fundamental tool providing support for this business strategy. According to reports provided by Gartner [4], company leading in information technology research, the value of the world's CRM market is forecasted to reach over 20 billion dollars in contrast to 2011 where revenues were projected to total $\$ 16.5$ billion. The evaluation was based on annual revenues of world leading providers of those systems. This proves the great interest in CRM by the both software industry and its customers. Moreover, together with the recent popularity growth of social networking interest in customer relationship management applications took on a new form.

\subsection{Social CRM}

The CRM philosophy is strongly associated with the concept of Social Capital, which can be defined as "the concept related to socioeconomy, based on the values that result from social network" [3]. In turn, Social Network is a set relationships and connections of a non-economic nature such as knowledge, competences or trust. In the last few years this topic was discussed very often after the growth of interest in blogging and the success of such a services as Facebook, Flickr and Twitter. Social services of this kind are the new type of media called Social Media. New studies show that today they have acquired a strategic importance for many companies. Linking social media with CRM systems is certainly the next step in their development. As a response to changing media trends, marketing researchers developed a new branch of CRM systems called Social CRM (SCRM or sCRM), oriented on the use of Social Media services. The definition of Social Customer Relationship Management [2] is as follows: 
"Social CRM is a philosophy and a business strategy, supported by a technology platform, business rules, processes, and social characteristics, designed to engage the customer in a collaborative conversation in order to provide mutually beneficial value in a trusted and transparent business environment. [...]".

As one can see the definitions of CRM and sCRM are very close with a difference in technology use, process conception and ways of interaction with the customer.

\section{Consensus system}

According to [6] consensus theory arisen in social science and has a root in choice theory. In general the consensus from a set $X$ (alternatives, objects), being a subset of a universe $U$, is a subset of $U$ (not of $X$ as in choice theory). The task of the consensus method is to determine a version of knowledge which best reflects given versions.

A consensus system in a CRM system is described by set of events resulting from customer activity. The events are investigated by agents whose job it is to make forecasts of customer behaviour based on those events. Each event is described by a set of attributes and their values. Events can be classified into groups on different subjects in the CRM system. Since agents may speak differently about the same subject, it is necessary to establish a consensus for such a situation. The consensus system $C S$ is defined as following quadruple [5]:

$$
C S=\langle A, X, P, Z\rangle
$$

where: $A$ - a finite set of attributes, which includes a special attribute Agent; each attribute $a \in A$ has a domain $V_{a}$ (a finite set of elementary values) such that values of a are subsets of $V_{a}$; values of attribute Agent are 1-element sets, which identify the agents.

$X$ - a finite set of consensus carriers, $X=\left\{\prod\left(V_{a}\right): a \in A\right\}$.

$P$ - a finite set of relations on carriers from $X$, each relation is of some type $T$ (for $T \subseteq A$ and Agent $\in T$ ).

$Z$ - a finite set of propositional calculus, for which the model is a relation system $(X, P)$

Set $X$ represents all objects which appear in the CRM system, in the context of behaviour prediction, $P$ represents events of user activity as properties of those objects from $X$ and their relations, and $Z$ represents conditions which have to be satisfied by relations from $P$.

In order to solve a consensus problem for supporting customer behaviour prediction in sCRM systems we decomposed the whole task into the following subproblems in the form of subsequent parts: (i) presenting knowledge scope by describing its elements, (ii) defining knowledge carriers in a form of agents, (iii) determining the structure of knowledge by defining its attributes and values, relations and conditions, (iv) defining conflict situations for previously defined relations (v) defining conflict profiles for conflict situations, (vi) determining consensus and defining distance functions 
which allow us to resolve conflicts for subjects of different situations, (vii) elaborating the consensus determination algorithm.

\subsection{Knowledge scope}

There are several concepts associated directly with CRM and sCRM which are considered as key structural elements of knowledge about customers. First of all, in CRM systems the information is gathered from the beginning of customer-company contact, often before the person actually becomes a customer. Such an identified, potential client is called a lead. Each client and lead is also connected with some opportunityestimated monetary value associated with an business event, for example acquiring a client or sending an offer.

A second group of knowledge structural elements are indicators which allow us to evaluate one of the most important components of the CRM strategy that is loyalty. The most useful factors in the context of customer behavior analysis are presented below.

The Recency Frequency Money $(R F M)$ factor [7]:

$$
R F M=(R \cdot \alpha)+(F \cdot \beta)+(M \cdot \gamma)
$$

where: $R$ - number of days since last purchase,

$\alpha$ - weight of last purchase,

$F$ - total number of purchases,

$\beta$ - weight of number of purchases,

$M$ - total value of purchases,

$\gamma$ - weight of the value of purchases.

The above indicator is very popular in CRM systems. It considers three measures: (a) when a customer has made his last purchase (Recency), (b) how often a customer buys a product (Frequency), (c) and how much money a customer usually spends on a product (Money). Each measure may have a different importance depending on the type of company and industry in which it operates. Therefore, each variable is assigned a weight, which the company adjusts individually. The second indicator is the Next Purchase Probability (NPP) [7]:

$$
N P P=\left(\frac{\alpha}{\beta}\right)^{n}
$$

where: $\alpha$ - number of days between first and last purchase,

$\beta$ - number of days taken into account in the historical client analysis,

$n$ - number of purchases in the entice historical period.

This indicator allows us to measure the probability that a customer will repeat his purchase in the near future. Because repeated purchases are one of the most important characteristics of loyalty, this factor is also very important in CRM. The last indicator useful in measuring loyalty in CRM is customer LifeTime Value (LTV) [7]:

$$
L T V=\alpha+\beta
$$


where:

$\alpha$ - annual profit from sales of products to the customer,

$\beta$ - number of years of customer-company relation.

The above index takes into account two fundamental measures of describing loyalty. These are customer life cycle expressed as the number of years of mutual relations, and profit from sales which is the most evident measure because it reflects company's financial results.

The last group of knowledge structural elements corresponds to components related to Social CRM. It contains information on client activity in the sphere of the most popular worldwide social networking websites. This includes: customer interest on Facebook (things that a client likes), and number of followers on Twitter. Follower is a person who follows another person's comments in a form of short messages.

\subsection{Knowledge carriers}

The knowledge carriers about customer behaviour are agents. According to our research conducted in [1], their knowledge is is stored in synaptic weights of Artificial Neural Network, based on a set of profile characteristics associated with the activities.

Customer profilse allow us to differentiate clients on the basis of their individual set of attributes. It contains basic information about a client, such as age, gender, city etc., and extended information such as favourite categories of products or opportunities associated with the customer. In addition, the profile also includes properties related to Social Media (interests on Facebook, followers on Twitter) and indicators which enable us to measure the level of customer loyalty such as RFM, NPP and LTV.

Customer activities concern elements which define his behaviour. From the perspective of the CRM philosophy, customer behaviour is a vector consisting of product categories, complaints, opportunities associated with a client or facts, defining potential customer as a lead.

Collected in this way knowledge is used to predict customer behaviour based on appropriate questions about the particular action. For the purpose of this paper, a neural network is trained for each customer separately and thus every agent forecasts different behaviour for the same customer. The task of this paper is to choose a knowledge version which best reflects the versions given by different agents.

\subsection{Knowledge structure}

All opinions on future behaviour elaborated by agents concern each customer separately. Knowledge about each client is composed of events of customer activity represented by attributes and their values, relations and conditions on those attributes.

\subsubsection{Attributes and values}

Knowledge of individual agents concerns $R F M, N P P$ and $L T V$ indicators (equations $2,3,4)$ which reflect the level of customer loyalty in the CRM system. Attributes 
Facebook and Twitter are common features of sCRM systems. The first one concerns the ID numbers of "like" positions as the topics of interests in the Facebook service. The second one is the number followers on Twitter. Every customer can purchase a product in some category, which is another possible attribute of client knowledge representation. The last attribute is value which holds monetary value for various relations, presented in the next subsection. Based on the above the possible attributes of customer behaviour are as follows:

$$
A=\{\text { Agent }, R F M, N P P, L T V, \text { Facebook, Twitter, Category, Value }\}
$$

Based on the above equation the consensus carriers $X$ resulting values of above attributes are as follows:

$$
X=\left\{\prod\left(V_{\text {Agent }}\right), \prod\left(V_{R F M}\right), \prod\left(V_{N P P}\right), \ldots, \prod\left(V_{\text {Value }}\right)\right\}
$$

where:

$V_{\text {Agent }}=\left\{a_{1}, a_{2}, a_{3}, \ldots, a_{n}\right\}$

$V_{R F M}=[1,+\infty]$

$V_{N P P}=[0,1]$

$V_{L T V}=[1,+\infty]$

$V_{\text {Facebook }}=[0,+\infty]$

$V_{\text {Twitter }}=[0,+\infty]$

$V_{\text {Category }}=\left\{c_{1}, c_{2}, c_{3}, \ldots, c_{n}\right\}$

$V_{\text {Value }}=[1,+\infty]$

\subsubsection{Relations and conditions}

We distinguish three fundamental to CRM systems relation groups which are based on three different aspects of customer activity. Those relation groups concern purchases, opportunities and leads. Because subsequent events of customer behaviour represent states of customer activity, knowledge in our consensus system for CRM is composed only of positive opinions carried by different agents. Therefore, the relations below are also composed only of positive knowledge:

$$
P=\{\text { Purchase, Opportunity, Lead }\}
$$

where: Purchase, Opportunity, Lead are the following types of relations:

Purchase : \{Agent, RFM, NPP, LTV, Facebook, Twitter, Category, Value $\}$

Opportunity : \{Agent, Facebook, Twitter, Category, Value $\}$

Lead : \{Agent, Facebook, Twitter, Category\}

For example we interpret a tuple $\left\langle\left\{a_{1}\right\},\{300\},\{0.5\},\{600\},\{2,5\},\{50\},\left\{c_{4}\right\},\{100\}\right\rangle$ of relation Purchase in the following way: first agent $a_{1}$ predicts that in the future, a particular consumer will buy product in category $c_{4}$ for the price of 100 , factors RFM, NPP, LTV will change to the following values: 300,0.5,600, consumer will like products with ID 2 and 5 and will have 50 followers on Twitter. Tuple 
$\left\langle\left\{a_{1}\right\},\{2,3\},\{100\},\left\{c_{1}, c_{2}\right\},\{400\}\right\rangle$ of relation Opportunity means that according to agent $a_{1}$ in the future, a particular consumer will make an opportunity (or become an opportunity in the case of a lead) worth 100 units and and will be connected with given Facebook like positions, Twitter followers or product category. Tuple $\left\langle\left\{a_{1}\right\},\{2\},\{1000\},\left\{c_{3}\right\}\right\rangle$ of the last relation Lead means that $a_{1}$ forecasts a lead on given interests, followers and category.

The above relations have to satisfy following conditions:

$$
\begin{aligned}
& Z=\{ \\
& (\operatorname{Purchase}(a, r, n, l, f, t, c, v)) \Rightarrow(\neg \operatorname{Lead}(a, f, t, c)), \\
& (\operatorname{Lead}(a, f, t, c)) \Rightarrow(\text { Opportunity }(a, f, t, c, v)) \text {, } \\
& (\text { Purchase }(a, r, n, l, f, t, c, v) \wedge r>300) \Rightarrow(\operatorname{Opportunity}(a, f, t, c, v)) \text {, } \\
& (\operatorname{Purchase}(a, r, n, l, f, t, c, v) \wedge n>0.7) \Rightarrow(\operatorname{Opportunity}(a, f, t, c, v)) \text {, } \\
& (\operatorname{Purchase}(a, r, n, l, f, t, c, v) \wedge l>1000) \Rightarrow(\operatorname{Opportunity}(a, f, t, c, v)) \text {, } \\
& (\text { Purchase }(a, r, n, l, f, t, c, v) \wedge t>10) \Rightarrow(\operatorname{Opportunity}(a, f, t, c, v)) \\
& \text { \} }
\end{aligned}
$$

The first condition means that according to agent $a$ if a person made a purchase it may not be a lead. The second condition states that every lead on interests $f$, followers $t$ and categories $c$ creates an opportunity on the same interests, followers and categories. The last three conditions mean that every purchase with $R F M$ higher than 300, NPP higher than 0.7, and $L T V$ higher that 1000 create an opportunity with the same $r, n, l, f, t, c$ and value $v$. Similarly, we create an opportunity if a client's Twitter followers is greater than 10 .

\subsection{Conflict situations}

A conflict situation is formed on the basis of different opinions of agents on specific event subjects which relate to customer activity in CRM system. Therefore, a conflict situation consists of: (i) relations which describe types of the event, (ii) the subject of a conflict on which agents make their forecasts, (iii) and the content of the conflict which comprises an agent's information on a given subject. An opinion of a single agent on the conflict subject is presented with a single tuple. Based on the above assumptions and regarding to [5] we define a conflict situation $s$ as:

$$
s=\langle P, A \rightarrow B\rangle
$$

where $A$ a represents conflict subject and $B$ the content of the conflict.

Customer behaviour prediction is based on data collected by Social CRM systems and concerns events described by relations $P$ (equation 7 ) of type Purchase, Opportunity, Lead. The conflict subjects for these events are categories 
of products that allow us to determine specific behaviour for a given event. The content of the conflicts are the CRM indicators and data on client activity in the sphere of social services. The information they carry allows us to establish a consensus for given conflict. Based on the above for every relation $P$ we define the following conflict situations:

$$
\begin{aligned}
& s_{1}=\langle\text { Purchase, Category } \rightarrow\{R F M, N P P, \text { LTV, Facebook, Twitter, Value }\}\rangle \\
& s_{2}=\langle\text { Opportunity, Category } \rightarrow\{\text { Facebook, Twitter, Value }\}\rangle \\
& s_{3}=\langle\text { Lead, Category } \rightarrow\{\text { Facebook, Twitter, Category }\}\rangle
\end{aligned}
$$

The above conflict situations make it possible to forecast a customer's purchase in a product category, the category of a customer's opportunity, and the category on which lead will be converted to customer. In the tables below we present examples of the defined above conflict situations (for better readability we skip the curly brackets when there is only one value in the set).

Table 1

Example of conflict situation $s_{1}$.

\begin{tabular}{c|c|cccccc} 
Agent & Category & RFM & NPP & LTV & Facebook & Twitter & Value \\
\hline$a_{1}$ & $c_{3}$ & 300 & 0.7 & 600 & $\{2,5\}$ & 1 & 80 \\
$a_{2}$ & $\left\{c_{1}, c_{2}\right\}$ & 320 & 0.7 & 710 & $\{1,5\}$ & 3 & 100 \\
$a_{3}$ & $c_{1}$ & 250 & 0.5 & 600 & $\emptyset$ & $\emptyset$ & 50 \\
$a_{4}$ & $\left\{c_{1}, c_{2}\right\}$ & 280 & 0.8 & 650 & $\{2,5\}$ & 1 & 100 \\
$a_{5}$ & $c_{1}$ & 310 & 0.6 & 600 & $\{2,5,7\}$ & 11 & 50
\end{tabular}

The conflict situation presented in Table 1 is created by seven different agents where each of them predicts different purchase possibilities of a single client. Agents $a_{6}$ and $a_{7}$ did not make any opinion on this event. The RFM, NPP, LTV indicators vary not only depending on purchase value and number of predicted purchases but also on time taken into account in an agent's forecast. Facebook and Twitter attributes also differ depending on agents parameters for a client's historical data analysis. A category's value estimation depends on a number of products and category average price.

Table 2

Example of conflict situation $s_{2}$.

\begin{tabular}{c|c|ccc} 
Agent & Category & Facebook & Twitter & Value \\
\hline$a_{1}$ & $c_{3}$ & 5 & $\emptyset$ & 50 \\
$a_{2}$ & $\left\{c_{1}, c_{2}\right\}$ & $\{1,5\}$ & 3 & 100 \\
$a_{4}$ & $\left\{c_{1}, c_{2}\right\}$ & $\{2,5\}$ & 1 & 100 \\
$a_{5}$ & $c_{1}$ & $\{2,5,7\}$ & 11 & 50 \\
$a_{6}$ & $\left\{c_{1}, c_{3}\right\}$ & $\{2,3\}$ & 5 & 100
\end{tabular}


The conflict situation in Table 2 presents opinions on a client's opportunity event and it is created by the same group of agents as in the case of the first situation. Forecasts of purchase events from the previous table made by agents $a_{2}, a_{4}, a_{5}$ were automatically translated to this event. The reason is that mentioned agents respectively conformed to condition number $3(r>300), 4(n>0.7)$ and $6(t>10)$ from the condition set $Z$ (Equation 8). Afterwards all seven agents made their forecasts on opportunity but only agents $a_{1}$ and $a_{6}$ presented their opinions.

Table 3

Example of conflict situation $s_{3}$.

\begin{tabular}{c|c|cc} 
Agent & Category & Facebook & Twitter \\
\hline$a_{6}$ & $c_{3}$ & 5 & $\emptyset$ \\
$a_{7}$ & $\left\{c_{1}, c_{3}\right\}$ & $\{1,2,3\}$ & 30
\end{tabular}

Conflict situation in Table 3 presents predictions concerning possible activities of leads. For this event only two of all seven agents could make a forecast because of the condition number 1 from set $Z$ which states that if an agent made a prediction of purchase for a particular client then he cannot make prediction of a lead for the same client, since after purchase every lead is considered as consumer.

\subsection{Conflict profiles}

In order to determine consensus from given conflict situations we firstly need to specify conflict profiles for every situation. A conflict profile is defined on subjects of conflict situations and it is represented as a set of different versions of knowledge (positive) about the same element of the real world - in our case events in the CRM environment. For each conflict subject $e \in$ Category we determine conflict profiles profile $(e)$ which contain opinions of agents on a given subject. The definition of conflict profile is as follows [5]:

$$
\text { profile }(e)=\left\{r_{B \cup\{\text { Agent }\}}:(r \in P) \wedge\left(e \prec r_{A}\right)\right\}
$$

The subject of every defined earlier conflict situation is the Category attribute. Based on presented in Table 1, Table 2 and Table 3 examples of conflict situations the resulting from them profiles illustrated in the following tables.

The subjects extracted from conflict situation type $s_{1}$ (Purchase event) are presented in Table 1 are $c_{1}, c_{2}$ and $c_{3}$. For every extracted subject we create separate profile, so that the whole dataset is sorted according to categories as Table 4 illustrates. We derive conflict profiles of situations $s_{2}$ (Opportunity event) and $s_{3}$ (Lead event) in the same manner. Resulting structures are presented in Table 5 and Table 6 , respectively.

Each profile represents positive knowledge on category subjects for presented conflict situations. With such a knowledge representations it is possible to determine consensus. 


\section{Table 4}

Example of conflict profiles for Purchase event.

\begin{tabular}{c||c|cccccc} 
Category & Agent & RFM & NPP & LTV & Facebook & Twitter & Value \\
\hline$c_{1}$ & $a_{2}$ & 320 & 0.7 & 710 & $\{1,5\}$ & 3 & 100 \\
$c_{1}$ & $a_{3}$ & 250 & 0.5 & 600 & $\emptyset$ & $\emptyset$ & 50 \\
$c_{1}$ & $a_{4}$ & 280 & 0.8 & 650 & $\{2,5\}$ & 1 & 100 \\
$c_{1}$ & $a_{5}$ & 310 & 0.6 & 600 & $\{2,5,7\}$ & 11 & 50 \\
\hline \hline$c_{2}$ & $a_{2}$ & 320 & 0.7 & 710 & $\{1,5\}$ & 3 & 100 \\
$c_{2}$ & $a_{4}$ & 280 & 0.8 & 650 & $\{2,5\}$ & 1 & 100 \\
\hline \hline$c_{3}$ & $a_{1}$ & 300 & 0.7 & 600 & $\{2,5\}$ & 1 & 80
\end{tabular}

Table 5

Example of conflict profiles for Opportunity event.

\begin{tabular}{c||c|ccc} 
Category & Agent & Facebook & Twitter & Value \\
\hline$c_{1}$ & $a_{2}$ & $\{1,5\}$ & 1 & 100 \\
$c_{1}$ & $a_{4}$ & $\{2,5\}$ & 1 & 100 \\
$c_{1}$ & $a_{5}$ & $\{2,5,7\}$ & 11 & 50 \\
$c_{1}$ & $a_{6}$ & $\{2,3\}$ & 5 & 100 \\
\hline \hline$c_{2}$ & $a_{2}$ & $\{1,5\}$ & 1 & 100 \\
$c_{2}$ & $a_{4}$ & $\{2,5\}$ & 1 & 100 \\
\hline \hline$c_{3}$ & $a_{1}$ & 5 & $\emptyset$ & 50 \\
$c_{3}$ & $a_{6}$ & $\{2,3\}$ & 5 & 100
\end{tabular}

Table 6

Example of conflict profiles for Lead event.

\begin{tabular}{c||c|ccc} 
Category & Agent & Facebook & Twitter & Category \\
\hline$c_{1}$ & $a_{7}$ & $\{1,2,3\}$ & 30 & $\left\{c_{1}, c_{3}\right\}$ \\
\hline \hline$c_{3}$ & $a_{6}$ & 5 & $\emptyset$ & $c_{3}$ \\
$c_{3}$ & $a_{7}$ & $\{1,2,3\}$ & 30 & $\left\{c_{1}, c_{3}\right\}$
\end{tabular}

\subsection{Consensus and distance function}

Referring to [5] the consensus of profile(e) on subject $e \in$ Category for situation $s=\langle P, A \rightarrow B\rangle$ is represented by tuple $C(s, e)$ of type $A \cup B$, which satisfies the logical formulas from set $Z$. Based on the above the consensus definition of a situation $s$ is as follows:

$$
C(s)=\{C(s, e): e \in \text { Category }\}
$$

According to Equation 14 the consensus of situation $s$ is a set of consensuses of every conflict profile of this situation. In order to establish consensus $C(s)$ distance functions are used. Those functions allow us to resolve conflicts for subjects for different situations. To do so, for every conflict situation we need to create resulting from it conflict profile. The distance function is used for measuring the distance between 
value sets $V_{a}$ of attributes $V$ for individual profiles. Each profile profile $(e)$ is formed on the basis of conflict subjects $e$ of conflict situation $s$. For predefined situations $s_{1}, s_{2}, s_{3}$ we use the following distance function [6]:

$$
\rho(X, Y)=\frac{1}{2 \operatorname{card}\left(V_{a}\right)-1} \sum_{z \in V_{a}} \operatorname{Part}(X, Y, z)
$$

where:

$\operatorname{Part}(X, Y, z)=1$ for every $z \in X \div Y$

$\operatorname{Part}(X, Y, z)=0$ for every $z \in X \cap Y$

$\operatorname{Part}(X, Y, z)=0$ for every $z \in V_{a} \backslash(X \cup Y)$

For the above formula function $\operatorname{card}(X)$ returns the cardinality of set $X$. The function described in Equation 15 is reflecting element shares in the distance. This kind of function is based on determining the value of shares of each element of set $V_{a}$ in the distance between two subsets of this set [6].

\subsection{Consensus determination algorithm}

Presented in this section algorithm allows us to determine consensus for Purchase, Opportunity, and Lead events describing the activity of a single customer or potential customer in a sCRM system. Because knowledge about above the activities is based on forecasts of different agents, the consensus of agent opinions represents the prediction of customer behaviour. Based on definitions presented in this paper the algorithm for consensus determination is as follows:

\section{Algorithm 1:}

Input: Set of conflict situation tuples $S=\left\{\left\langle s_{11}, s_{21}, s_{31}\right\rangle,\left\langle s_{12}, s_{22}, s_{32}\right\rangle, \ldots,\left\langle s_{1 n}, s_{2 n}, s_{3 n}\right\rangle\right\}$.

Output: Set of consensus tuples $C=\left\{\left\langle C\left(s_{11}\right), C\left(s_{21}\right), C\left(s_{31}\right)\right\rangle, \ldots,\left\langle C\left(s_{1 n}\right), C\left(s_{2 n}\right), C\left(s_{3 n}\right)\right\rangle\right\}$.

1: $\quad C \leftarrow \emptyset$

2: $\quad$ for $s$ Tuple $\in S$ do

3: $\quad C(s) \leftarrow\langle\rangle$

4: $\quad$ for $s \in$ sTuple do

5: $\quad C(s, e) \leftarrow \emptyset$

6: $\quad$ for $e \in$ Category and Category $\in s$ do

7: $\quad$ for prediction $\in \operatorname{Agent}(e)$ do

8: $\quad$ profile $(e) \leftarrow$ profile $(e) \cup$ prediction

9: $\quad$ end

10: $\quad$ for subjectSet $\in$ profile $(e)$ do

11: $\quad$ for $V_{b} \in B$ do

12: $\quad \rho_{V_{b}} \leftarrow \rho_{V_{b}} \cup \rho\left(V_{b}\right.$, profile $\left.(e)_{\text {subjectSet }+1, V_{b}}\right)$

13: $\quad$ end 


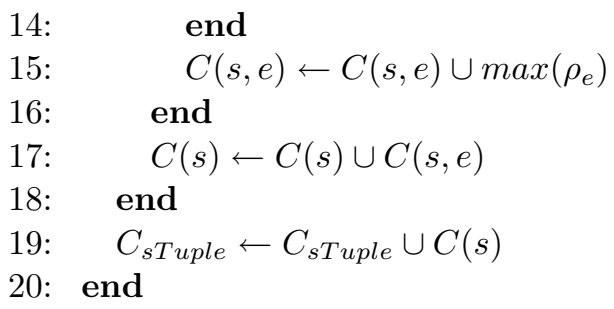

The input of Algorithm 1 is set of $n$ tuples $\left\langle s_{1 n}, s_{2 n}, s_{3 n}\right\rangle$, each representing conflict situations of $n$-th customer. The output is set of $n$ tuples $\left\langle C\left(s_{1 n}\right), C\left(s_{2 n}\right), C\left(s_{3 n}\right)\right\rangle$, each representing behaviour prediction of $n$-th customer, for all three events. For every conflict situation $s$ (lines 4-18) we extract conflict subjects $e$ (lines 6-16) from which we create a set of conflict profiles profile $(e)$ based on subsequent predictions on subject $e$ (lines 7-9). Secondly, in lines 10-14, we iterate over every opinion set subjectSet from created conflict profiles, in order to measure the distance between subsequent elements $V_{b}$ of current and next subjectSet. Resulting distances are appended to distance set $\rho_{V_{b}}$ (line 12). The maximal value from the set of distances for current subject is considered as its consensus and it is appended to the profile consensus $C(s, e)$ (line 15). Next, we append $C(s, e)$ of every profile to $C(s)$. In the end $C(s)$ of every event type is appended to the output set.

\section{Conclusions and future work}

Social Customer Relationship Management systems represent new area in the field of CRM which together with a rapid development of Social Networks and Social Media has acquired strategic importance for many companies. The application of such analysis meets the expectations of customers through a better match to their needs.

In this paper we presented intelligent tools for customer behaviour prediction in Social CRM systems which use a consensus approach in order to resolve different conflict situations. We define conflict situation as contradictory forecasts of behaviour of a particular customers or potential customers predicted by different agents. Agents are considered as knowledge carriers which store knowledge about customer behaviour in synaptic weights of ANN. In sCRM systems we distinguished three events: Purchase, Opportunity and Lead. Those events represent the actual targets of behaviour forecasts. Every event is described by attributes, values, relations and conditions which allow us to give their definitions. In order to establish consensus $C(s)$ distance functions were presented. Those functions allow us to resolve conflicts for subjects of different situations. To do so, for every conflict situation we presented how to create resulting from them conflict profiles.

The presented consensus system as a tool for predicting customer behaviour is based on clear examples that illustrate how conflict situations and resulting from them profiles are created. The entire tool is summarized by the presented algorithm which 
allows us to make behaviour predictions of many customers by establishing consensus. The presented approach is preceded by an analysis of a CRM and sCRM concepts.

In future work we consider using methods, which allow us to check consensus susceptibility. Such a feature helps to avoid computing in situations where consensus cannot be determined. Another issue is to elaborate method for finding common consensus for every $C(s, e)$ holding profile consensuses. Last and the most important tasks in the close future is to provide experimental comparisons of customer behaviour prediction using different distance functions.

\section{References}

[1] Czyszczoń A., Zgrzywa A.: Zastosowanie sztucznych sieci neuronowych do przewidywania zachowania klientów w systemie CRM, pp. 61-72. Wydawnictwo WTN, xviii edition, 2011.

[2] Greenberg P.: CRM at the Speed of Light: Social CRM Strategies, Tools, and Techniques for Engaging Your Customers, 4th ed. McGraw-Hill, 2010.

[3] Grzanka I.: Kapitał społeczny w relacjach z klientami. CeDeWu, 2009.

[4] Gartner Inc..: Gartner press release. http://www.gartner.com/it/section.jsp, February 2012.

[5] Nguyen N. T.: Consensus system for solving conflicts in distributed systems. Information Sciences, 147(14):91 - 122, 2002.

[6] Nguyen N.T.: Advanced Methods for Inconsistent Knowledge Management (Advanced Information and Knowledge Processing). Springer-Verlag New York, Inc., Secaucus, NJ, USA, 2008.

[7] Urban W., Siemieniako D.: Lojalność klientów. Modele, motywacja i pomiar. Wydawnictwo Naukowe PWN, Warszawa, 2008.

\section{Affiliations}

\section{Adam Czyszczoń}

Wrocław University of Technology, Faculty of Computer Science and Management, Wrocław, Poland, adam.czyszczon@pwr.wroc.pl

Aleksander Zgrzywa

Wrocław University of Technology, Faculty of Computer Science and Management, Wrocław, Poland, aleksander.zgrzywa@pwr.wroc.pl

Received: 30.04 .2012

Revised: 2.07 .2012

Accepted: 3.09 .2012 